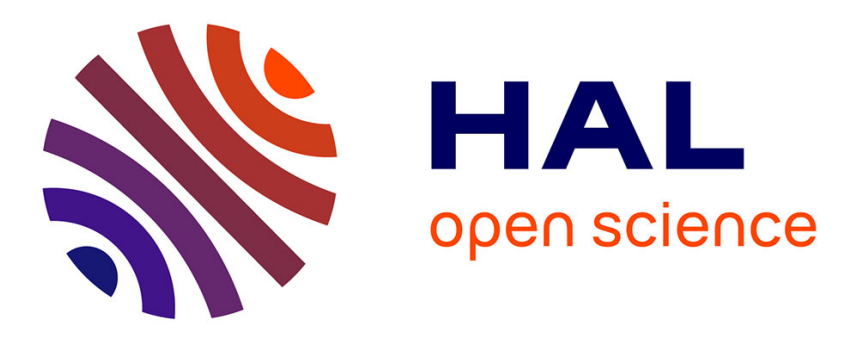

\title{
Jumping ability: A theoretical integrative approach
}

Pierre Samozino, J.B. Jean-Benoît Morin, Frédérique Hintzy, Alain Belli

\section{To cite this version:}

Pierre Samozino, J.B. Jean-Benoît Morin, Frédérique Hintzy, Alain Belli. Jumping ability: A theoretical integrative approach. Journal of Theoretical Biology, 2010, 264 (1), pp.11. 10.1016/j.jtbi.2010.01.021 . hal-00574834

\section{HAL Id: hal-00574834 https://hal.science/hal-00574834}

Submitted on 9 Mar 2011

HAL is a multi-disciplinary open access archive for the deposit and dissemination of scientific research documents, whether they are published or not. The documents may come from teaching and research institutions in France or abroad, or from public or private research centers.
L'archive ouverte pluridisciplinaire HAL, est destinée au dépôt et à la diffusion de documents scientifiques de niveau recherche, publiés ou non, émanant des établissements d'enseignement et de recherche français ou étrangers, des laboratoires publics ou privés. 


\section{Author's Accepted Manuscript}

Jumping ability: A theoretical integrative approach

Pierre Samozino, Jean-Benoît Morin, Frédérique Hintzy, Alain Belli

PII: $\quad$ S0022-5193(10)00033-0

DOI: doi:10.1016/j.jtbi.2010.01.021

Reference: YJTBI 5843

To appear in: $\quad$ Journal of Theoretical Biology

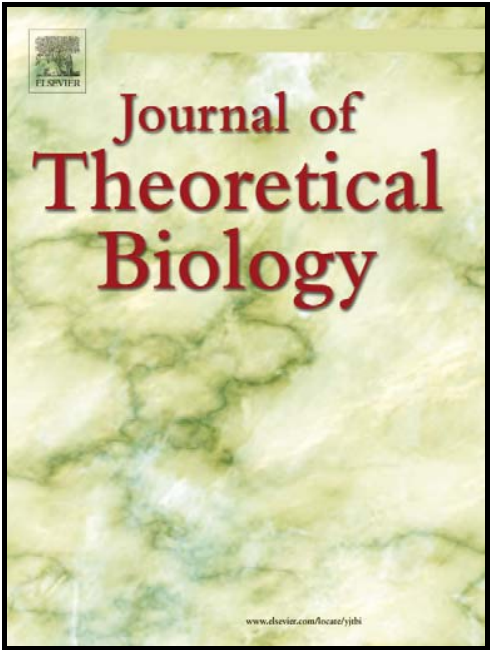

www.elsevier.com/locate/yjtbi

Received date: $\quad 20$ July 2009

Revised date: $\quad 15$ January 2010

Accepted date: 19 January 2010

Cite this article as: Pierre Samozino, Jean-Benoît Morin, Frédérique Hintzy and Alain Belli, Jumping ability: A theoretical integrative approach, Journal of Theoretical Biology, doi:10.1016/j.jtbi.2010.01.021

This is a PDF file of an unedited manuscript that has been accepted for publication. As a service to our customers we are providing this early version of the manuscript. The manuscript will undergo copyediting, typesetting, and review of the resulting galley proof before it is published in its final citable form. Please note that during the production process errors may be discovered which could affect the content, and all legal disclaimers that apply to the journal pertain. 


\section{Title}

J umping ability: a theoretical integrative approach

\section{Authors}

Pierre SA M OZIN O, J ean-B enoît M ORIN, Frédérique HINTZY , A lain B ELLI

\section{Institution}

University of Lyon, F-42023, Saint Etienne, France

Laboratory of Exercise Physiology (EA 4338), F-42000, Saint-Etienne, France.

\section{Correspondance}

Pierre SA M OZINO ( $\square)$

L aboratoire de Physiologie de I'Exercice

Université de Saint-Étienne, M édecine du sport et M yologie - CHU B ellevue

42055 Saint-Étienne Cedex 02, France

Tel: +33477120733

E-mail: pierre.samozino@univ-st-etienne.fr

\section{Key words}

1) maximal jump

2) explosive movement

3) force - velocity relationship

4) performance

5) lower extremity 


\section{Summary}

A theoretical integrative approach is proposed to understand the overall mechanical characteristics of lower extremities determining jumping ability. This approach considers that external force production during push-off is limited by mechanical constraints imposed by both movement dynamics and force generator properties, i.e. lower extremities characteristics. While the velocity of the body depends on the amount of external force produced over the push-off, the capabilities of force production decrease with increasing movement velocity, notably for force generators driven by muscular contraction, such as lower extremities of large animals during jumping from a resting position. Considering the circular interaction between these two mechanical constraints, and using simple mathematical and physical principles, the proposed approach leads to a mathematical expression of the maximal jump height an individual can reach as a function of only three integrative mechanical characteristics of his lower extremities: the maximal force they can produce $\left(\bar{F}_{0}\right)$, the maximal velocity at which they can extend under muscles action $\left(\bar{v}_{0}\right)$ and the distance of force production determined by their usual extension range $\left(h_{P O}\right)$. These three integrative variables positively influence maximal jump height. For instance in humans, a 10\% variation in $\bar{F}_{0}, \bar{v}_{0}$ or $h_{P O}$ induces a change in jump height of about 10 to $15 \%, 6$ to $11 \%$ and 4 to $8 \%$, respectively. The proposed theoretical approach allowed to isolate the basic mechanical entities through which all physiological and morphological specificities influence jumping performance, and may be used to separate the very first macroscopic effects of these three mechanical characteristics on jumping performance variability. 


\section{List of abbreviations}

CM body center of mass

$m$ body mass (in $\mathrm{kg}$ )

$g$ gravitational acceleration $\left(9.81 \mathrm{~m} \cdot \mathrm{s}^{-2}\right)$

$h \quad$ jump height corresponding to the vertical distance covered by the $\mathrm{CM}$ during the aerial phase (in $\mathrm{m}$ )

$W_{T} \quad$ total external mechanical work produced by lower extremities during push-off (J)

$h_{P O} \quad$ vertical push-off distance determined by lower extremities extension range (in $\mathrm{m}$ )

$v_{\text {TO }} \quad$ CM vertical velocity at take-off (in $\mathrm{m} \cdot \mathrm{s}^{-1}$ )

$\bar{F} \quad$ mean vertical external force developed over push-off (relative to body mass, in N.kg ${ }^{-1}$ )

$\bar{v} \quad$ mean vertical CM velocity over push-off (in $\mathrm{m}_{\mathrm{s}}^{-1}$ )

$\bar{F}_{0} \quad$ theoretical maximal value of $\bar{F}$ that lower extremities can produce during one extension at a theoretical null $\bar{v}$ (relative to body mass, in N. $\mathrm{kg}^{-1}$ )

$\bar{v}_{0} \quad$ theoretical maximal value of $\bar{v}$ at which lower extremities can extend during one extension under the influence of muscles action in a theoretical unloaded condition (in $\left.\mathrm{m} \cdot \mathrm{s}^{-1}\right)$

$t_{P O} \quad$ push-off time (in s)

$v_{\text {TOmax }}$ maximal CM vertical take-off velocity a given animal can reach $\left(\mathrm{m} \cdot \mathrm{s}^{-1}\right)$

$h_{\max } \quad$ maximal jump height a given animal can reach $(\mathrm{m})$ 


\section{Introduction}

M aximal jumps aim to cover the highest vertical and/or horizontal distance from a large acceleration of the body during one quick legs extension. A ccording to $\mathrm{N}$ ew ton's laws of motion, the acceleration of a body during a push-off phase depends on the resultant force applied on it (fundamental principle of dynamics), itself resulting from the force developed by the individual (animal or human) on the environment (law of reciprocal actions).

Consequently, accelerating one's body mass from a resting position to reach the highest jumping performance depends on the production of a large amount of external force during one quick lower extremities extension. Identifying the overall mechanical characteristics of the force generator (i.e. the lower extremities) allowing to maximise this force production over the whole push-off phase could increase the understanding of the mechanical factors limiting jumping performance.

The characteristics of lower extremities related to maximal jumps have been mainly studied through the physiological or morphological traits statistically related to jumping ability (e.g. muscle mass, limbs length, muscle fibre types, joint moment arms), that are the phenotypic expressions of the overall mechanical characteristics of lower extremities involved in performance (see reviews by James et al., 2007; M arsh, 1994). The relationship between jumping performance and overall mechanical characteristics of lower extremities, such as force or power output capabilities, has only been explored through correlations, and in only few studies on humans, which did not reach a consensus (Driss et al., 1998; Y amauchi and Ishii, 2007). Such statistical approaches present the risk of interaction between covariant parameters making uncertain the direct causal effect between dependent and independent variables, though related in some cases by significant correlations. Hence, the large number of biological features that may act on jumping ability (together or independently) make the 
overall understanding of lower extremities characteristics involvement in such abilities difficult on the sole basis of experimental data.

For these reasons, theoretical approaches have been proposed to better understand the mechanical factors affecting jumping ability. The best examples are forward simulation models that integrate a large amount of morphological, physiological or neuromuscular parameters (A lexander, 1995; B obbert and V an Soest, 1994; Pandy and Zajac, 1991). These models allowed to answer several questions that could not be solved experimentally, such as optimal muscular coordination strategies or effects of different jumping techniques (e.g. A lexander, 1995; B obbert and Casius, 2005; Pandy and Zajac, 1991). However, they do not make it possible to isolate the basic mechanical characteristics of lower extremities determining the ability to accelerate the body. Indeed, the numerous inputs used do not represent the overall mechanical characteristics of the force generator, but specific biological features that affect these mechanical characteristics, several of them acting on the same entity. Other theoretical framew orks have explored these overall mechanical characteristics affecting jumping ability through the investigation of the mechanical requirements of maximal jumps. Indeed, jumping ballistic and dynamic analyses allow one to identify the mechanical outputs of push-off determining jumping performance at best. For instance, jumping performance has been mathematically shown to be proportional to the mechanical work produced during pushoff (A lexander, 2003a; Emerson, 1985) or to the mean power developed bef ore take-off (M arsh, 1994). These mathematical equations let think that maximal jumping performances are determined by the capability of lower extremities to produce work or power. However, other authors supported the idea that impulsive action performance (such as jumping) do not depend on muscles ability to generate power, but rather on their capability to produce impulse (A damson and Whitney, 1971; Knudson, 2009; Winter, 2005). Even if this distinction may seem minor, it reveals the difficulty in identifying the mechanical characteristics of the force 
generator that maximise jumping performance from the sole mechanical outputs related to performance. Whether it is impulse, work or power that determines performance, the question arises as to which mechanical characteristic(s) of lower extremities allow(s) to maximise it. Further, analyses of the mechanical behaviour of an accelerating body show that all the mechanical outputs characterising push-off dynamics are closely connected (M arsh, 1994; M inetti, 2002; W akai and Linthorne, 2005). From a theoretical framew ork, M inetti showed that an increase in the force applied to a mass made the other mechanical outputs of push-off vary: decrease in push-off duration and increase in power and velocity for instance (Minetti, 2002). Regarding the latter point, and for most of large animals in which skeletal muscles form the basis of the force generator, the increase in movement velocity is associated with a decrease in force production capability (Hill, 1938). Hence, the higher the force production, the faster the movement, and the lower the force production capability. Consequently, take-off velocity, and so jumping performance, depends on the force production of lower extremities over push-off, itself depending on mechanical characteristics of the force generator, themselves depending on push-off velocity. Due to this circular interaction between force generator capabilities and the dynamics of an accelerated body, the mechanical constraints imposed by both force generator and movement dynamics should be taken into account to identify the mechanical characteristics related to maximal jumps.

The aim of this study is therefore to propose an alternative theoretical integrative approach (i) to identify the overall mechanical characteristics of lower extremities determining maximal jumping ability and (ii) to quantify their respective influence by means of the simplest mathematical equation(s) possible. This theoretical framew ork considers the mechanical constraints of both movement and force generator limiting jumping performance. Only vertical jumping will be considered here since it is an excellent system to investigate jumping abilities. 


\section{Theoretical background}

The mechanical constraints imposed by both force generator characteristics and movement dynamics on jumping ability are mainly expressed through the interaction between the amount of force produced and the centre of mass (CM ) velocity over the whole push-off. The aim of the following sections is to understand how these two mechanical constraints affect the interdependence of force production and movement velocity.

\subsection{Mechanical constraints imposed by movement dynamics}

J ump height $(h)$, which corresponds here to the $\mathrm{CM}$ aerial vertical displacement, is dependent on the $\mathrm{CM}$ vertical velocity at take-off $\left(v_{\mathrm{TO}}\right)$, according to this basic ballistic equation: $h=\frac{v_{T O}{ }^{2}}{2 g}$

This section analyses the push-off phase through the dynamics of the body CM, aiming, with a mathematical approach, to understand how $v_{T O}$ depends on force production. The push-off is considered here in its wholeness, and not studied moment-by-moment. Let consider here a push-off phase during which a body of mass $m$ is accelerated along a vertical direction. L ower extremities are represented by a linear force generator. The CM velocity (and hence kinetic energy) being null before push-off, the total external mechanical work $\left(W_{T}\right)$ done by lower extremities during push-off is equal to the variation in the mechanical energy of the body, which is the sum of potential and kinetic energies:

$W_{T}=\frac{1}{2} m v_{T O}^{2}+m g h_{P O}$

with $g$ the gravitational acceleration and $h_{\mathrm{PO}}$ the vertical push-off distance corresponding to the extension range of lower extremities. Furthermore, $W_{T}$, developed by lower extremities 
during push-off, is also equal to the product of $h_{P O}$ times the mean vertical external force produced over push-off :

$$
\bar{F}=\frac{W_{T}}{m h_{P O}}
$$

with $\bar{F}$ the mean vertical external force produced over push-off expressed relative to body mass (in N. $\mathrm{kg}^{-1}$ ). In this form, $\bar{F}$ has the dimension of an acceleration and would correspond to the component of the $\mathrm{CM}$ acceleration induced by the lower extremities propulsive force. Substituting (2) in this equation gives:

$$
\bar{F}=\frac{v_{T O}^{2}}{2 h_{P O}}+g
$$

From equation (4), $v_{T O}$ may be expressed as:

$$
v_{T O}=\sqrt{2 h_{P O}(\bar{F}-g)}
$$

This relationship between $v_{T O}, \bar{F}$ and $h_{P O}$ represents the mechanical constraints imposed by movement dynamics of an accelerated body. Hence, $v_{T O}$ depends on the mean force produced over push-off, on body mass (since $\bar{F}$ is expressed relative to body mass) and on the distance through which the force is produced (that is the extension range of lower extremities). The general relationship between $v_{T O}$ and $\bar{F}$ is presented in Fig. 1 for different values of $h_{P O}$ during a vertical push-off. Each line represents, for different values of $h_{\mathrm{PO}}$, the force-velocity conditions ( $\bar{F}-v_{T O}$ conditions) allowed by movement dynamics, each $\bar{F}-v_{T O}$ condition corresponding to one jump height (obtained from equation (1)). Jumping performance increases when $h_{P O}$ increases and/or when $\bar{F}$ increases, $\bar{F}$ and $v_{T O}$ changing concomitantly in a specific way depending on $h_{P O}$ (equation (5)). However, this concomitant increase in $\bar{F}$ and $v_{T O}$ is limited by the mechanical characteristics of the force generator. Indeed, force 
production capabilities are well known to depend on velocity for force generators composed by skeletal muscles.

\section{- Figure 1 around here -}

\subsection{Mechanical constraints imposed by the force generator}

Let consider here only individuals propelling themselves by the direct action of muscles, that is most of large animals (A lexander, 1995). The mechanical characteristics of the force generator are thus mainly related to the mechanical properties of muscles. The mechanical limits of skeletal muscles capabilities, and notably of the contractile elements, are represented by an inverse force-velocity relationship (F-V relationship), well documented in isolated muscles (Hill, 1938) as well as in entire lower extremities musculature during various types of human movement (R ahmani et al., 2001; Sargeant et al., 1981; V andewalle et al., 1987). This overall inverse relationship, described as linear for pluri-articular movements (B osco et al., 1995; Rahmani et al., 2001; V andewalle et al., 1987; Y amauchi and Ishii, 2007), accounts for the decrease in the maximal capability for entire lower extremities to generate force with increasing movement velocity. Such linear F-V relationships have widely been showed from mean values of force $(\bar{F})$ and velocity $(\bar{v})$ over limbs extension, and have often been defined by two values: the theoretical maximal $\bar{F}$ that lower extremities can produce over one extension $\left(\bar{F}_{0}\right.$, expressed relative to body mass, in N. $\left.\mathrm{kg}^{-1}\right)$ and the theoretical maximal $\bar{v}$ at which lower extremities can extend during one extension under the influence of muscle action $\left(\bar{v}_{0}\right.$ in $\left.\mathrm{m} . \mathrm{s}^{-1}\right)$. Graphically, $\bar{F}_{0}$ and $\bar{v}_{0}$ correspond to the force-axis and velocity-axis intercepts of the F-V curve, respectively. Thus, during a dynamic maximal effort, such as the push-off phase of a maximal jump, the maximal $\bar{F}$ that can be produced may be expressed as a function of $\bar{v}$ : 
$\bar{F}=\bar{F}_{0}-\frac{\bar{F}_{0}}{\bar{v}_{0}} \bar{v}$

During a maximal jump with a null starting velocity, $\bar{v}$ and $v_{T O}$ are closely related, as shown in the following demonstration. From Newton's second law of motion, the change in momentum of a body is proportional to the net impulse impressed on this body, and applies along the straight line on which this impulse is impressed. A pplying the impulse-momentum relationship to the considered push-off phase with null starting velocity gives:

$v_{T O}=(\bar{F}-g) \cdot t_{P O}$

with $t_{P O}$ the push-off phase duration. From equations (4) and (7), $t_{P O}$ may be expressed as: $t_{P O}=\frac{2 h_{P O}}{v_{T O}}$

The mean CM vertical velocity $\bar{v}$ over the whole push-off is the ratio between $h_{P O}$ and $t_{P O}$, and thus may be obtained from equation (8):

$\bar{v}=\frac{v_{T O}}{2}$

This equation is only based on the impulse-momentum relationship, and so does not assume that the CM acceleration is constant during push-off, as it was done by Bosco et al. (1983) to show the same result.

\section{- Figure 2 around here -}

\subsection{Determination of the maximal $v_{T O}$ that can be reached}

Considering both the mechanical constraints imposed by the movement itself ( $v_{T O}$ increasing with $\bar{F}$, equation (5)) and those imposed by the force generator (force production capability decreasing with increasing velocity, equation (6)) allows to determine the maximal $\bar{F}$ that can be produced during a vertical push-off, maximising $v_{T O}$ and $h$. Fig. 2 presents these two 
kinds of mechanical constraints acting on force production during a vertical push-off for a given human-like $h_{P O}(0.4 \mathrm{~m})$. The grey area under the F-V relationship (dashed line) represents the whole $\bar{F}-\bar{v}$ conditions that lower extremities musculature can develop, the $\mathrm{F}$ $V$ relationship being the $\bar{F}-\bar{v}$ conditions corresponding to maximal efforts. The solid line represents, as in Fig. 1, the $\bar{F}-v_{T O}$ conditions allowed by the movement dynamics, higher jumping performance being reached when $\bar{F}$ increases. Consequently, the maximal value of $v_{T O}\left(v_{T O \text { max }}\right)$ that can be reached is achieved in the push-off condition allowed by both force generator maximal capabilities and movement dynamics. Such on optimal condition corresponds graphically to the couple of force and velocity values at the two lines intersect. Consequently, the force and velocity values characterising this optimal condition have to be a valid set of solutions for the system formed by equations (5), (6) and (9), the unknown variables being $\bar{F}, \bar{v}$ and $v_{T O}$. Solving this system of equations, which includes a non-linear equation (equation (5)), gives two quadratic equations each including $\bar{F}$ or $v_{T O}$. The quadratic equation including $v_{T O}$ has only one real positive solution for $v_{T O}\left(v_{\text {TOmax }}\right)$ that may be expressed as:

$v_{\text {TOmax }}=h_{P O}\left(\sqrt{\frac{\bar{F}_{0}^{2}}{4 \overline{\bar{V}}_{0}^{2}}+\frac{2}{h_{P O}}\left(\bar{F}_{0}-g\right)}-\frac{\bar{F}_{0}}{2 \bar{v}_{0}}\right)$

From equation 1 , the maximal jump height that can be reached $\left(h_{\max }\right)$ may be expressed as:

$h_{\max }=\frac{h_{P O^{2}}}{2 g}\left(\sqrt{\frac{\bar{F}_{0}{ }^{2}}{4 \bar{v}_{0}{ }^{2}}+\frac{2}{h_{P O}}\left(\bar{F}_{0}-g\right)}-\frac{\bar{F}_{0}}{2 \bar{v}_{0}}\right)^{2}$

From these computations, $h_{\max }$ that could be conceivable respecting the mechanical constraints of both movement and force generator depends only on $\bar{F}_{0}, \bar{v}_{0}$ and $h_{P O}$, which are 
three mechanical entities characterising the lower extremities overall capabilities. Note that equation (11) is true for $\bar{v}_{0}>0, h_{P O}>0$ and $\bar{F}_{0}>g$.

\section{Results}

The influences of $\bar{F}_{0}, \bar{v}_{0}$ and $h_{P O}$ on $h_{\max }$ were analysed from equation (11). First, a sensitivity analysis was performed plotting the relative variations of $h_{\max }$ against the relative variations of $\bar{F}_{0}, \bar{v}_{0}$ and $h_{P O}$, each parameter being studied separately (Fig. 3). The reference values of the three parameters corresponded to human-like values $\left(\bar{F}_{0}=30 \mathrm{~N} \cdot \mathrm{kg}^{-1}, \bar{v}_{0}=3 \mathrm{~m} \cdot \mathrm{s}^{-1}\right.$ and $h_{P O}=0.4 \mathrm{~m}$; e.g. B osco et al., 1995; Rahmani et al., 2001), but the range of variations considered allowed a wide variety of large animals to be concerned. M aximal jump height increases when $\bar{F}_{0}, \bar{v}_{0}$ or $h_{P O}$ increase, and it is more sensitive to $\bar{F}_{0}$ than to $\bar{v}_{0}$ or $h_{P O}$. Indeed, changes in $h_{\max }$ with $\bar{F}_{0}$ variations are higher than those induced by $\bar{v}_{0}$ variations, themselves higher than those induced by $h_{P O}$ variations: a $10 \%$ change in $\bar{F}_{0}$ leads to a $\sim 10$ to $15 \%$ change in $h_{\max }$, while the same $10 \%$ change in $\bar{v}_{0}$ and $h_{P O}$ leads to changes in $h_{\max }$ ranging from 6 to $11 \%$ and from 4.5 to $7.5 \%$, respectively. $N$ ote that these relative influences were calculated from reference values corresponding to human characteristics, and may be different for other reference values.

\section{- Figure 3 around here -}

Second, absolute influences of $\bar{F}_{0}, \bar{v}_{0}$ and $h_{P O}$ on $h_{\max }$ variations are presented in Fig. 4 . The range of $\bar{F}_{0}, \bar{v}_{0}$ and $h_{P O}$ values were chosen to correspond to most of large animals, jumping by means of direct muscles contraction (A lexander 1995). The positive influence of the three parameters on $h_{\max }$ is confirmed for a wide range of values. M oreover, the shape of contours 
shows that the influence of each parameter is enhanced by the increase in the two other parameters.

\section{- Figure 4 around here -}

Considering the few values of $\bar{F}_{0}$ (between 25 and $50 \mathrm{~N} \cdot \mathrm{kg}^{-1}$ ) and $\bar{v}_{0}$ (between 1.5 and $8 \mathrm{~m} . \mathrm{s}$ ${ }^{1}$ ) reported in previous human studies (Rahmani et al., 2004; Rahmani et al., 2001; Y amauchi and Ishii, 2007) and estimating $h_{P O}$ between 0.35 and $0.45 \mathrm{~m}, h_{\max }$ values obtained range from $\sim 0.2$ to $\sim 0.6 \mathrm{~m}$, which is in line with human vertical jumping performance (Fig. 4 , the maximal values of each parameter being unlikely obtained by a same given subject). Further, the validity of equation (11) has been confirmed by experimental data comparing actual and predicted values of $h_{\max }$ in human vertical jumps (unpublished personal data).

\section{Discussion}

This study proposes a novel mathematical approach to identify the mechanical characteristics of lower extremities involved in maximal jumping ability and to quantify their respective influence on this ability. Considering the mechanical constraints of both movement and force generator that limit the force production during push-off, this integrative approach leads to a mathematical expression of the maximal jump height an individual can reach $\left(h_{\max }\right)$ as a function of only three mechanical entities that represent overall mechanical characteristics of lower extremities: the theoretical maximal dynamic force that can be generated over one lower extremities extension $\left(\bar{F}_{0}\right)$, the theoretical maximal velocity at which the lower extremities can extend under the influence of muscles contraction in unloaded conditions $\left(\bar{v}_{0}\right)$, and the extension range of the lower extremities determining the distance over which force can be generated $\left(h_{P O}\right)$. 


\subsection{Limits of the theoretical integrative approach}

The theoretical developments proposed to express $h_{\max }$ as a function of only three mechanical characteristics of lower extremities required many simplifying assumptions that could limit their application. First, since the mechanical properties of the force generator have been characterised by an inverse F-V relationship, the present form of equation (11) only applies to animals propelling themselves by the direct action of muscles. Further, the linearity of the F$\checkmark$ relationship of the entire lower extremities musculature, as well as the extrapolation of $\bar{F}_{0}$ and $\bar{v}_{0}$, is well admitted for human lower limbs extension (Rahmani et al., 2001; Y amauchi and Ishii, 2007), but has not been thoroughly investigated in animals, mainly for obvious practical reasons. It is notew orthy that $\bar{F}_{0}$ and $\bar{v}_{0}$ are two purely theoretical values and have to be considered as target values towards which maximal capabilities of the force generator tend. Hence, $\bar{F}_{0}$ and $\bar{v}_{0}$ have to be understood as the "force" and "velocity" maximal capabilities of the entire lower extremities. Other reasonable assumptions have been made, such as those related to the application of Newton's laws to a whole body considered as a system or the neglected air resistance which only affects very small animals jump height (body mass below $0.5 \mathrm{~g}$, Scholz et al., 2006a). M oreover, it was supposed that the CM vertical displacement during push-off corresponded to the extension range of the lower extremities (i.e. $h_{P O}$ ), whereas the actual relative position of the $\mathrm{CM}$ within the body shifts slightly downwards due to this lower extremities extension. This overestimation has nevertheless minor effects on mechanical outputs estimation during a jumping push-off (Samozino et al., 2008). The main limit of the proposed approach remains in the relationship, existing in some cases, between $h_{P O}$ and the two parameters characterising the force generator $\left(\bar{F}_{0}\right.$ and $\left.\bar{v}_{0}\right)$. Indeed, $\bar{F}_{0}$ and $\bar{v}_{0}$, as mean values representing maximal force and velocity over the whole push-off, are specific to the lower extremities range of extension $\left(h_{P O}\right)$. For a given individual, a change in 
$h_{P O}$ (induced for instance by a change in starting position) may lead to variations in $\bar{F}_{0}$ and $\bar{v}_{0}$ due to the effects of both muscles force-length relationships and changes in joint moment arms during extension. Hence, $\bar{F}_{0}$ and $\bar{v}_{0}$ correspond to the maximal force and velocity capabilities of lower extremities for a given extension range, i.e. for a given $h_{P O}$. Consequently, the effect of intra-individual variations in $h_{P O}$ on $h_{\max }$ has to be cautiously considered since $h_{P O}$ variations may involve changes in $\bar{F}_{0}$ and $\bar{v}_{0}$. Therefore, comparisons of jumping ability for a given individual at two different instants (e.g. before and after training) require to keep $h_{P O}$ constant (M inetti, 2002; Zamparo et al., 2002). However, for inter-individual or inter-species comparisons, the considered $h_{\mathrm{PO}}$ has to correspond to the optimal or usual one for each individual, which allows to investigate differences in maximal performance between individuals. In the following part of this discussion, when the effect of $h_{P O}$ on $h_{\text {max }}$ is considered, one should consider several individuals with different usual or optimal $h_{P O}$, and not the effect of intra-individual changes in $h_{P O}$.

\subsection{Jumping ability is explained by only three integrative mechanical}

\section{variables}

The originality of the proposed approach is to explain jumping performance from the fewest variables possible, which agrees for instance with A lexander's conception of mathematical models in biological systems: "the simpler the model, the clearer it is which of its characteristics are essential to the observed effect" (A lexander, 2003b). The whole point of explaining jumping performance by the fewest lower extremities mechanical characteristics possible is to isolate the basic mechanical entities through which all physiological and morphological specificities influence jumping performance. The three integrative variables $\left(\bar{F}_{0}, \bar{v}_{0}\right.$ and $\left.h_{P O}\right)$ identified in equation (11) do not correspond to phenotypic traits, but 
represent the overall mechanical characteristics of the entire lower extremities. This implies these variables to be integrative, and the explored resulting effects to be macroscopic. These three mechanical characteristics encompass all the morphological and physiological parameters previously put forward to explain the variability in jumping performance. For instance, $\bar{F}_{0}$ and $\bar{v}_{0}$ do not only correspond to intrinsic muscles properties, but are the resultant of all the biological features affecting the maximal force that can be developed during lower extremities extension and the maximal extension velocity, respectively. The positive influence of $\bar{F}_{0}$ on $h_{\max }$ is in line with studies relating jumping ability to muscle strength (A lexander, 1995; Cheng, 2008; M affiuletti et al., 2002; Scholz et al., 2006b; U grinowitsch et al., 2007; Y amauchi and Ishii, 2007) or animals hind limb muscle mass (Choi and Park, 1996; J ames and Wilson, 2008; J ames et al., 2007). Indeed, maximal muscle force, which is proportional to muscles cross-sectional areas (A lexander, 1985), logically increases with the amount of muscle mass involved in push-off. Other factors previously related to jumping performance directly affect $\bar{F}_{0}:$ a high rate of force development ( $V$ anezis and $L$ ees, 2005), an optimised motor unit recruitment (M affiuletti et al., 2002) or positions of muscles origin and insertion increasing torque and force production (E merson, 1985). Besides, it is worth noting that $\bar{F}_{0}$ represents a force normalised to body mass (expressed in equation (11) in $\mathrm{N} . \mathrm{kg}^{-1}$ ). Since larger animals are known to produce lower maximal forces relative to their body mass (and thus lower $\bar{F}_{0}$ ), $h_{\max }$ is logically influenced by animals body size, as previously reported (A lexander, 1985; J ames et al., 2007; Scholz et al., 2006a).

Similarly to $\bar{F}_{0}$, the positive influence of $\bar{v}_{0}$ on $h_{\max }$ is in accordance with studies showing that frog muscles used to power jumping are mainly composed of fast-twitch muscle fibres (Lutz et al., 1998; M arsh, 1994), and with studies relating jumping performance to muscle strength only at high movement velocity (Eckert, 1968). The effect of $\bar{v}_{0}$ on jumping 
performance has also been suggested through the positions of muscles insertion around joints, favouring in many cases the velocity of movement (Emerson, 1985). N euromuscular coordination strategies, widely presented as determinant for vertical jumping performance (Bobbert and V an Soest, 1994; Pandy and Zajac, 1991), are also integrated in the mechanical characteristics $\bar{F}_{0}$ and $\bar{v}_{0}$. The same is true for the positive effect of $h_{P O}$ on $h_{\max }$. Indeed, $h_{P O}$ includes numerous morphological characteristics that increase the range of motion and have previously been related to jumping performance, such as hind limb length (Choi and Park, 1996; Emerson, 1985; H arris and Steudel, 2002; J ames and Wilson, 2008), the number of joints and their angular range of motion (A lexander, 1995), or the height of the starting position (A lexander, 1995; Domire and Challis, 2007; Sel bie and Caldwell, 1996). Interestingly, James Gray al ready put forward that it was not the leg length itself that is advantageous in jumping, but the ability to extend the limb to a greater length (Gray, 1953).

\subsection{Respective influences of $\bar{F}_{0}, \bar{v}_{0}$ and $h_{P O}$ on $h_{\max }$}

The three mechanical characteristics $\bar{F}_{0}, \bar{v}_{0}$ and $h_{\mathrm{PO}}$, and all the above mentioned biological characteristics that positively influence them, have a positive effect on jumping ability. This is obvious when referring to Figs. 1 and 2 : increasing $h_{\max }$ may be achieved by shifting the F-V relationship of lower extremities to the right (i.e. increasing both $\bar{F}_{0}$ and $\bar{v}_{0}$ ) and/or by shifting the curve of the $\bar{F}-v_{T O}$ conditions allowed by movement dynamics upwards (i.e. increasing $h_{P O}$ ). Simulations performed with equation (11) show that $h_{\text {max }}$ is more dependent on $\bar{F}_{0}$ or $\bar{v}_{0}$ than on $h_{P O}$. As a result, maximal jump seems to be more limited by the mechanical characteristics of force generator (i.e. F-V relationship) than by movement dynamics depending on $h_{P O}$. When trying to differentiate the respective weight of $\bar{F}_{0}$ and $\bar{v}_{0}$, simulations performed from human-like reference values (sensitivity analysis in Fig. 3) seem 
to show that maximal jumping ability is more limited by $\bar{F}_{0}$ than by $\bar{v}_{0}$. Thus, force capabilities seem to represent the mechanical entity playing the most important role in jumping performance, at least in humans. The respective weight of either $\bar{F}_{0}$ or $\bar{v}_{0}$ on $h_{\max }$ seems to be enhanced with the increase in the other variables, as shown by the shape of contours in Fig. 4. Consequently, jump height of smaller animals, known to produce larger relative maximal force (A lexander, 1985), is more sensitive to $\bar{v}_{0}$ changes than larger animals jump height is, which agrees with A lexander's model simulations (A lexander, 1995). In the same way, in hypogravity conditions, in which the relative maximal force of an individual is higher than on the Earth, the influence of $\bar{v}_{0}$ on jump height would increase to the detriment of the influence of $\bar{F}_{0}$, which is in line with M inetti's conclusions (M inetti, 2002). The mathematical expression of jumping performance (equation (11)) provides a theoretical framework to separate the very first macroscopic effects of $\bar{F}_{0}, \bar{v}_{0}$ and $h_{P O}$ on jumping performance variability. A dopting the same reasoning as M inetti (2002), equation (11) could be used to separate the effects of intra-individual changes in force or velocity capabilities when a change in jumping performance is observed. Conversely, one could use equation (11) to predict jump height changes induced by expected modifications (positive or negative) in force generator capabilities, which may guide rehabilitation and/or training programs. On the other hand, such an integrative approach could also form a first stage to guide further explorations of underlying biomechanical and physiological mechanisms at lower levels of organization. For instance, a simple application of equation (11) makes it possible to explore the differences in jumping ability between humans and bonobos from data presented by Scholz et al (2006b). If $\bar{F}_{0}$ and $\bar{v}_{0}$ are similar, the observed difference of maximal jump height between the man $(0.34 \mathrm{~m})$ and the bonobo $(0.52 \mathrm{~m}$ estimated without arm movement effect) may be explained for $\sim 40 \%$ (i.e. $\sim 0.07 \mathrm{~m}$ ) by the difference in $h_{P O}(\sim 0.42 \mathrm{~m}$ for the 
man, $\sim 0.65 \mathrm{~m}$ for the bonobo from their optimal starting position). The remaining $\sim 60 \%$ of difference (i.e. $\sim 0.11 \mathrm{~m}$ ) may be then explained by better force generator characteristics ( $\bar{F}_{0}$ and/or $\bar{v}_{0}$ ), and notably, as argued by the authors, by a higher specific force (maximal force per unit muscle mass).

\subsection{Other strategies to improve jumping abilities}

In addition to biological adaptations, individuals also used specific techniques that allow higher maximal jump height by overcoming the muscular constraints. The most famous one in humans is the countermovement performed just before push-off, leading to 2 to $4 \mathrm{~cm}$ higher jumps (B obbert and Casius, 2005). This greater performance has been firstly explained by effects of elastic energy and myoelectrical potentiation (B osco et al., 1982), and more recently by the only fact that muscle active state develops during the preparatory downward movement, which involves a higher force at the beginning of push-off, and so improves the maximal force capability (B obbert and Casius, 2005). In Fig. 2, the improvement in jump height thanks to a countermovement may be graphically represented by a shift of the lower extremities $\mathrm{F}-\mathrm{V}$ relationship to the right, that is by an increase in $\bar{F}_{0}$ and/or $\bar{v}_{0}$ (Bosco and K omi, 1979; B osco et al., 1982). A nother strategy has been observed in small animals to face with morphological or muscular constraints, such as short hind limbs limiting $h_{P O}$ (Burrows and Sutton, 2008; J ames et al., 2007) or poor muscle maximum shortening velocity reducing $\bar{v}_{0}$ (A lexander, 1995; J ames et al., 2007). Such a jump technique, particularly described in insects (B ennet-Clark, 1975) and commonly called "catapult mechanism" (A lexander, 1995; James et al., 2007), allows animals to overcome skeletal muscles limits by storing elastic energy and releasing it suddenly, that is more quickly than what muscles alone could do (A lexander, 1995; B ennet-Clark, 1975; B urrows and Sutton, 2008). In many small insects, in 
which the whole amount of energy required to jump can be stored in elastic structures (B ennet-Clark, 1975), jumping performance is not limited by muscle contractile properties but rather by the mechanical characteristics of elastic energy storage and recoil. Considered as a linear spring, the series elastic elements can therefore be assumed to shorten at unlimited velocities (A lexander, 1995), the corresponding $\bar{v}_{0}$ value tending then to infinite. Simple computations (from data reported by B ennet-Clark (1975) and A lexander (1995)) may show that the maximal jump height a locust can reach using the catapult mechanism may be estimated to $\sim 50 \mathrm{~cm}$ while muscles contraction alone would lead to jumps not higher than 5 $\mathrm{cm}$. With the "catapult mechanism", the muscle F-V relationship is not a mechanical limit to jumping performance, which is in line with the low influence of the muscle maximal shortening velocity on the "catapult" jump height (A lexander, 1995). O ther animals, larger than insects, have been shown to also use "catapult-like" mechanisms to jump, namely frogs (e.g. James et al., 2007) or bushbabies (e.g. A erts, 1998). Even if the mechanical work done during push-off is provided by both elastic energy release and muscles contraction, this jump technique, also qualified as a "mechanical power amplifier" (A erts, 1998), largely improves jumping performance by increasing the "velocity" capabilities of the force generator.

\section{Conclusion}

This study proposed a new integrative approach leading to a mathematical expression of the maximal jump height an individual can reach from only three mechanical characteristics of its lower extremities: their maximal capability of force production, their maximal extension velocity and their usual extension range. This theoretical approach increases then the understanding of the mechanical factors limiting jumping performance. The main originality of this mathematical expression is to isolate the basic mechanical entities through which all physiological and morphological specificities influence jumping performance, and to quantify 
the respective influence of these entities on jumping ability. When exploring the inter-species, inter-individuals or intra-individual variability in jumping performance, this integrative expression may help to separate the very first macroscopic effects of these three lower extremities characteristics. Even if the proposed integrative approach applies to vertical jumps in large animals, it could be generalised and adapted to other types of maximal push-off or other force generators used to propel a given mass. 


\section{REFERENCES}

A damson, G.T., and W hitney, R.J ., 1971. Critical A ppraisal of J umping as a M easure of Human Power, in: V redenbregt, J. and W artenweiller, J., Eds.), Biomechanics II, V ol. 6.K arger, S., B asel, pp. 208-211.

A erts, P., 1998. V ertical jumping in galago senegalensis: the quest for an obligate mechanical power amplifier. Philos. Trans. R. Soc. Lond. B. Biol. Sci. 353, 1607-1620.

A lexander, R.M., 1985. The maximum forces exerted by animals. J. Exp. B iol. 115, 231-238.

A lexander, R.M., 1995. L eg design and jumping technique for humans, other vertebrates and insects. Philos. Trans. R. Soc. Lond. B. Biol. Sci. 347, 235-48.

A lexander, R.M., 2003a. Climbing and J umping, Principles of animal locomotion, Princeton University Press, pp. 146-165.

A lexander, R.M., 2003b. M odelling approaches in biomechanics. Philos. Trans. R. Soc. L ond. B. Biol. Sci. 358, 1429-35.

B ennet-Clark, H.C., 1975. The energetics of the jump of the locust Schistocerca gregaria. J . Exp. Biol. 63, 53-83.

Bobbert, M.F., and V an Soest, A.J ., 1994. Effects of muscle strengthening on vertical jump height: a simulation study. M ed. Sci. Sports Exerc. 26, 1012-20.

B obbert, M.F., and Casius, L.J., 2005. Is the effect of a countermovement on jump height due to active state development? M ed. Sci. Sports Exerc. 37, 440-6.

B osco, C., and K omi, P.V ., 1979. Potentiation of the mechanical behavior of the human skeletal muscle through prestretching. A cta Physiol. Scand. 106, 467-72.

B osco, C., L uhtanen, P., and K omi, P.V., 1983. A simple method for measurement of mechanical power in jumping. Eur. J. A ppl. Physiol. Occup. Physiol. 50, 273-82.

B osco, C., V iitasalo, J.T., K omi, P.V., and L uhtanen, P., 1982. Combined effect of elastic energy and myoelectrical potentiation during stretch-shortening cycle exercise. A cta Physiol. Scand. 114, 557-65.

B osco, C., B elli, A ., A strua, M., Tihanyi, J., Pozzo, R., K ellis, S., T sarpela, O., Foti, C., M anno, R., and Tranquilli, C., 1995. A dynamometer for evaluation of dynamic muscle work. Eur. J. A ppl. Physiol. Occup. Physiol. 70, 379-86.

B urrows, M ., and Sutton, G.P., 2008. The effect of leg length on jumping performance of short- and long-legged leafhopper insects. J. Exp. Biol. 211, 1317-25.

Cheng, K.B., 2008. The relationship between joint strength and standing vertical jump performance. J. A ppl. Biomech. 24, 224-33.

Choi, I., and Park, K., 1996. V ariations in take-off velocity of anuran amphibians: Relation to morphology, muscle contractile function and enzyme activity. Comp. Biochem. Physiol. 113A, 393-400.

Domire, Z.J ., and Challis, J.H., 2007. The influence of squat depth on maximal vertical jump performance. J. Sports Sci. 25, 193-200.

Driss, T., V andewalle, $H_{\text {., }}$ and $M$ onod, $H_{\text {., }}$ 1998. M aximal power and force-velocity relationships during cycling and cranking exercises in volleyball players. Correlation with the vertical jump test. J. Sports M ed. Phys. Fitness 38, 286-93. .

Eckert, H.M., 1968. A ngular velocity and range of motion in the vertical and standing broad jumps. Res. Q. 39, 937-42.

Emerson, S.B., 1985. J umping and L eaping, in: Hildebrand, M. E., et al., Eds.), Functional V ertebrate M orphology, Harvard university Press, Cambridge, pp. 58-72.

Gray, J., 1953. Jumping and Creaping, How animals move, Cambridge U niversity Press, pp. 69-88. 
Harris, M .A ., and Steudel, K ., 2002. The relationship between maximum jumping performance and hind limb morphology/physiology in domestic cats (F elis silvestris catus). J. Exp. Biol. 205, 3877-89.

Hill, A .V., 1938. The heat of shortening and the dynamic constants of muscle. Proc. R. Soc. Lond. B Biol. Sci. 126B, 136-195.

James, R.S., and Wilson, R.S., 2008. Explosive jumping: extreme morphological and physiological specializations of A ustralian rocket frogs (Litoria nasuta). Physiol. Biochem. Zool. 81, 176-85.

James, R.S., Navas, C.A., and Herrel, A ., 2007. How important are skeletal muscle mechanics in setting limits on jumping performance? J. Exp. Biol. 210, 923-33.

K nudson, D.V., 2009. Correcting the use of the term "power" in the strength and conditioning literature. J. Strength. Cond. Res. 23, 1902-8.

L utz, G.J., B remner, S., Lajevardi, N., Lieber, R.L., and Rome, L.C., 1998. Quantitative analysis of muscle fibre type and myosin heavy chain distribution in the frog hindlimb: implications for locomotory design. J. M uscle Res. Cell M otil. 19, 717-31.

M affiuletti, N .A ., Dugnani, S., Folz, M., Di Pierno, E., and M auro, F., 2002. Effect of combined electrostimulation and plyometric training on vertical jump height. $M$ ed. Sci. Sports Exerc. 34, 1638-44.

M arsh, R.L., 1994. Jumping ability of anuran amphibians. A dv. V et. Sci. Comp. M ed. 38B, 51-111.

M inetti, A .E., 2002. On the mechanical power of joint extensions as affected by the change in muscle force (or cross-sectional area), ceteris paribus. Eur. J. A ppl. Physiol. 86, 363-9.

Pandy, M .G., and Zajac, F.E., 1991. O ptimal muscular coordination strategies for jumping. J. Biomech. 24, 1-10.

Rahmani, A ., L ocatelli, E., and Lacour, J.R., 2004. Differences in morphology and force/velocity relationship between Senegalese and Italian sprinters. Eur. J. A ppl. Physiol. 91, 399-405.

Rahmani, A ., V iale, F., D alleau, G., and Lacour, J.R., 2001. Force/velocity and power/velocity relationships in squat exercise. Eur. J. A ppl. Physiol. 84, 227-32.

Samozino, P., M orin, J.B., Hintzy, F., and B elli, A., 2008. A simple method for measuring force, velocity and power output during squat jump. J. B iomech. 41, 2940-5.

Sargeant, A.J., Hoinville, E., and Y oung, A., 1981. M aximum leg force and power output during short-term dynamic exercise. J. A ppl. Physiol. 51, 1175-82.

Scholz, M.N., B obbert, M.F., and K noek van Soest, A.J., 2006a. Scaling and jumping: gravity loses grip on small jumpers. J. Theor. B iol. 240, 554-61.

Scholz, M.N., D'A out, K., B obbert, M .F., and A erts, P., 2006b. Vertical jumping performance of bonobo (Pan paniscus) suggests superior muscle properties. Proc. R. Soc. B 273, 2177-84.

Selbie, W.S., and Caldwell, G.E., 1996. A simulation study of vertical jumping from different starting postures. J. Biomech. 29, 1137-46.

U grinowitsch, C., Tricoli, V., Rodacki, A .L., B atista, M ., and Ricard, M .D., 2007. Influence of training background on jumping height. J. Strength. Cond. Res. 21, 848-52.

V andewalle, H., Peres, G., H eller, J., Panel, J., and M onod, H., 1987. Force-velocity relationship and maximal power on a cycle ergometer. Correlation with the height of a vertical jump. Eur. J. A ppl. Physiol. Occup. Physiol. 56, 650-6. .

$V$ anezis, A ., and L ees, A., 2005. A biomechanical analysis of good and poor performers of the vertical jump. Ergonomics 48, 1594-1603.

W akai, M ., and Linthorne, N.P., 2005. O ptimum take-off angle in the standing long jump. Hum M ov Sci 24, 81-96.

Winter, E.M ., 2005. J umping: Power or Impulse. M ed. Sci. Sports Exerc. 37, 523-4. 
Y amauchi, J., and Ishii, N., 2007. Relations between force-velocity characteristics of the knee-hip extension movement and vertical jump performance. J. Strength. Cond. Res. 21, 703-9.

Zamparo, P., M inetti, A.E., and di Prampero, P.E., 2002. Interplay among the changes of muscle strength, cross-sectional area and maximal explosive power: theory and facts. Eur. J. A ppl. Physiol. 88, 193-202. 


\section{FIGURES CAPTIONS}

\section{Figure 1}

Changes in the take-off velocity and jump height as a function of the mean force produced over vertical jump push-off for different push-off distances $\left(h_{P O}\right)$. Each line represents the Force-V elocity conditions allowed by movement dynamics during a given push-off. J ump height and take-off velocity are related by equation (1). Since force is expressed relative to body mass, the $\mathrm{x}$-axis also corresponds to the component of the CM acceleration induced by the propulsive force.

\section{Figure 2}

Theoretical representation of the mechanical constraints imposed by movement dynamics (continuous line) and lower extremities capabilities (dashed line) during a typical vertical jump. The continuous line represents the take-off velocity according to the mean force produced over push-off. The Force - V elocity conditions (mean force and mean velocity over push-off) allowed by lower extremities mechanical capabilities are represented for sub maximal efforts (grey area) and for maximal explosive effort (dashed line). Here, $h_{P O}, \bar{F}_{0}$ and $\bar{v}_{0}$ values are $0.4 \mathrm{~m}, 30 \mathrm{~N} \cdot \mathrm{kg}^{-1}$ and $3 \mathrm{~m} \cdot \mathrm{s}^{-1}$, respectively.

\section{Figure 3}

Sensitivity analysis: variations of the three low er extremities mechanical characteristics $\left(\bar{F}_{0}, \bar{v}_{0}\right.$ and $\left.h_{P O}\right)$ against the corresponding variations in maximal jump height. The reference values for the three mechanical characteristics are $30 \mathrm{~N} . \mathrm{kg}^{-1}, 3 \mathrm{~m} \cdot \mathrm{s}^{-1}$ and $0.4 \mathrm{~m}$ for $\bar{F}_{0}, \bar{v}_{0}$ and $h_{P O}$, respectively. 


\section{Figure 4}

Contour plots showing the influence of $\bar{F}_{0}, \bar{v}_{0}$ and $h_{P O}$ on the maximal jump height an animal can reach using its lower extremities musculature as force generator. Each plot shows the influence of $\bar{F}_{0}$ and $\bar{v}_{0}$ on maximal jump height for three values of $h_{P O}$ characterising different individuals. The contours show the jump height (in m). 


\section{FIGURES}

Figure 1

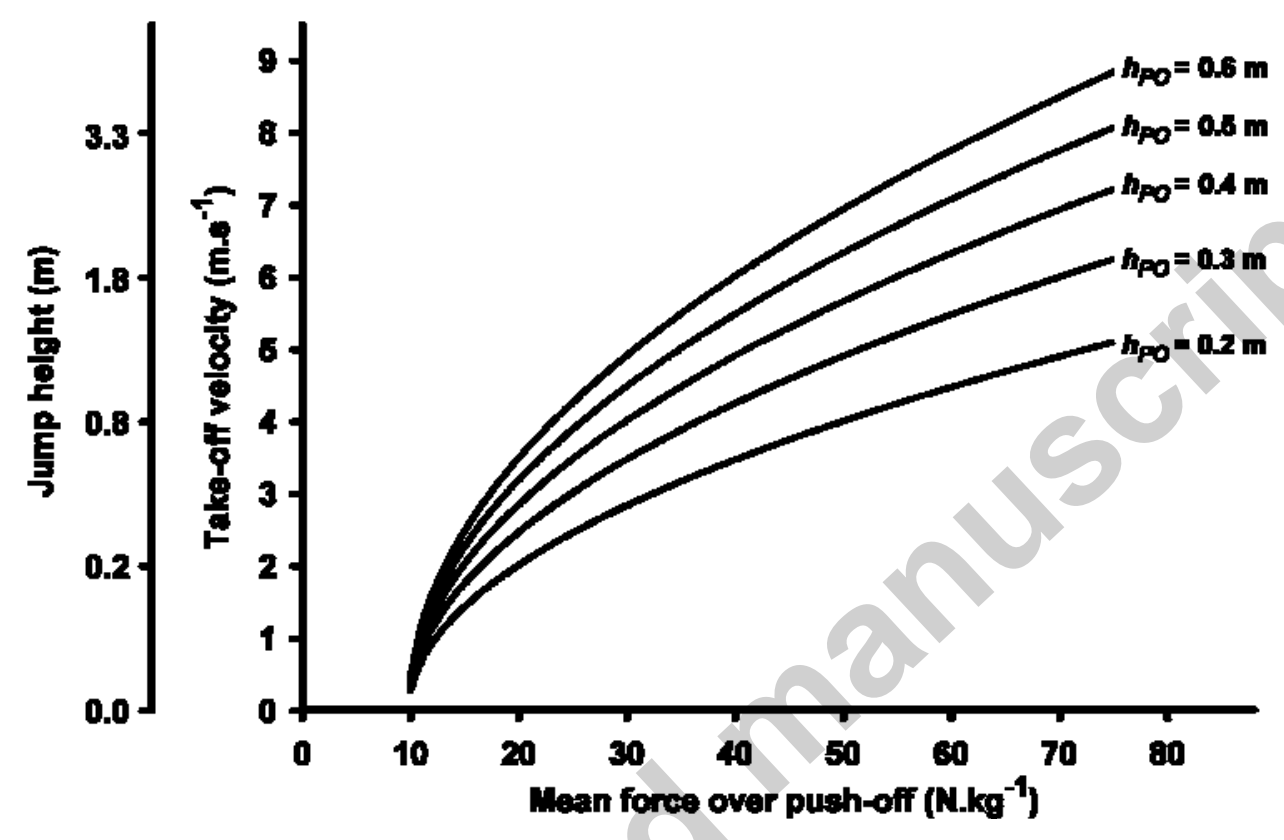


Figure 2

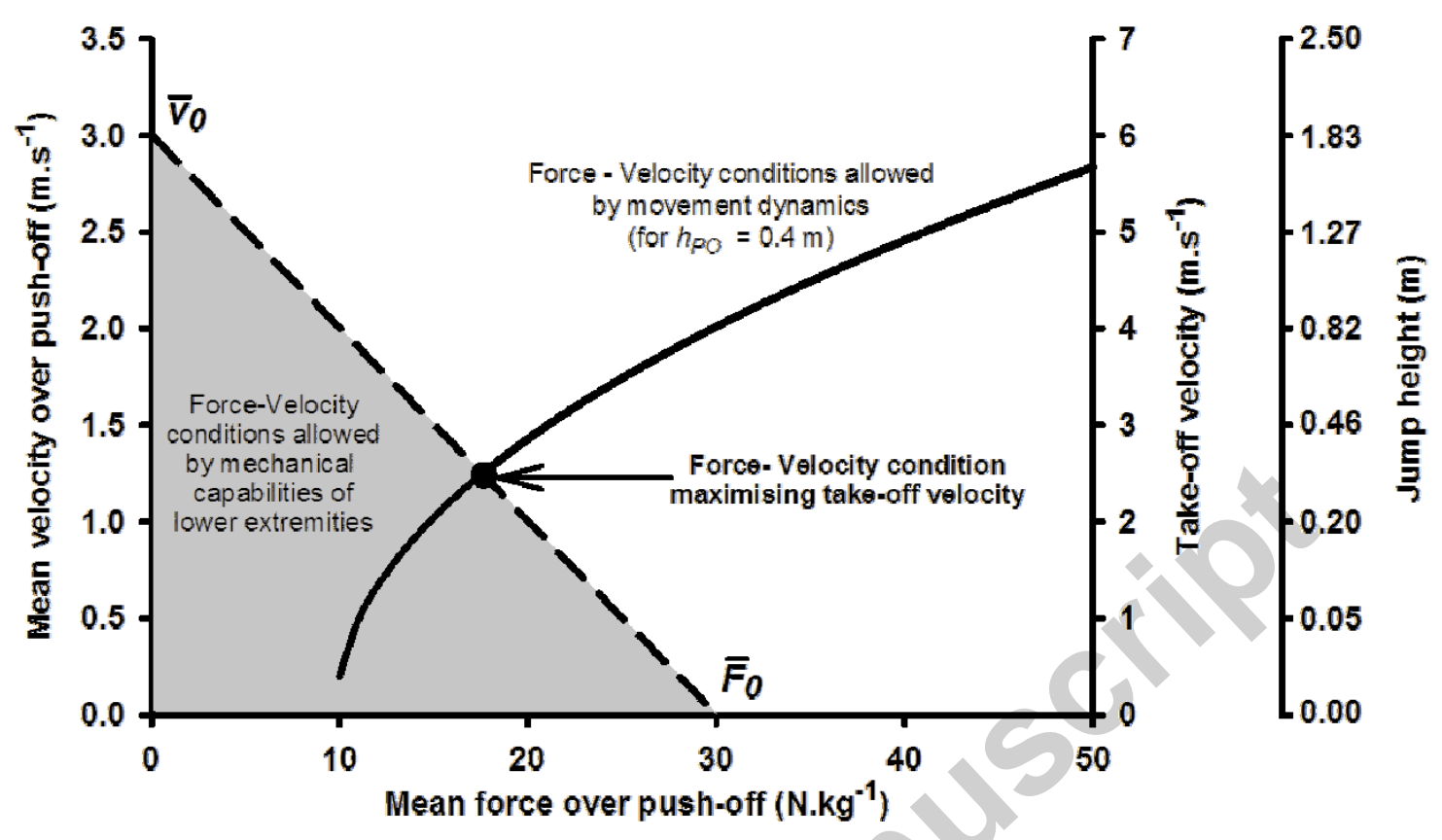


Figure 3

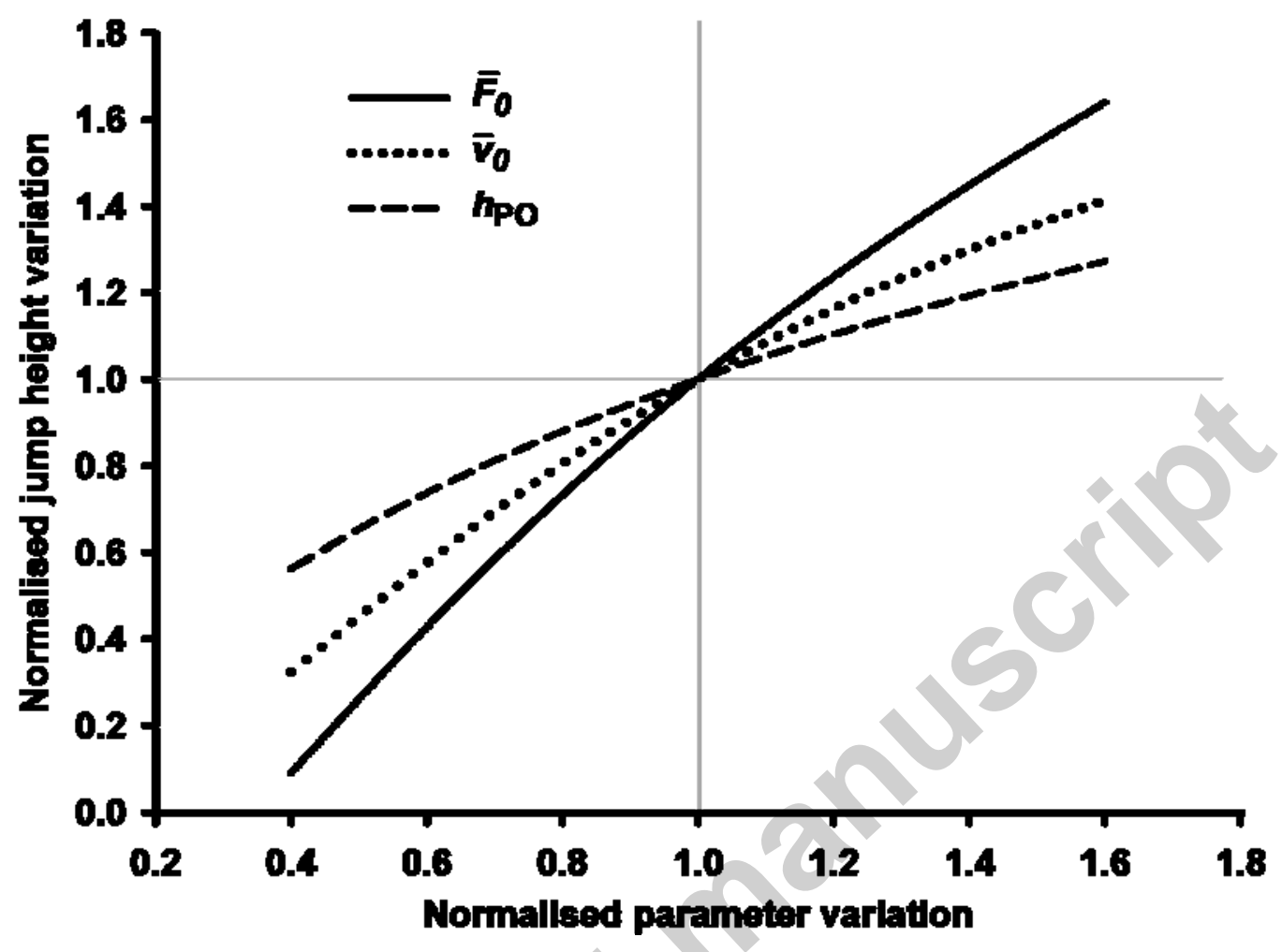


Figure 4

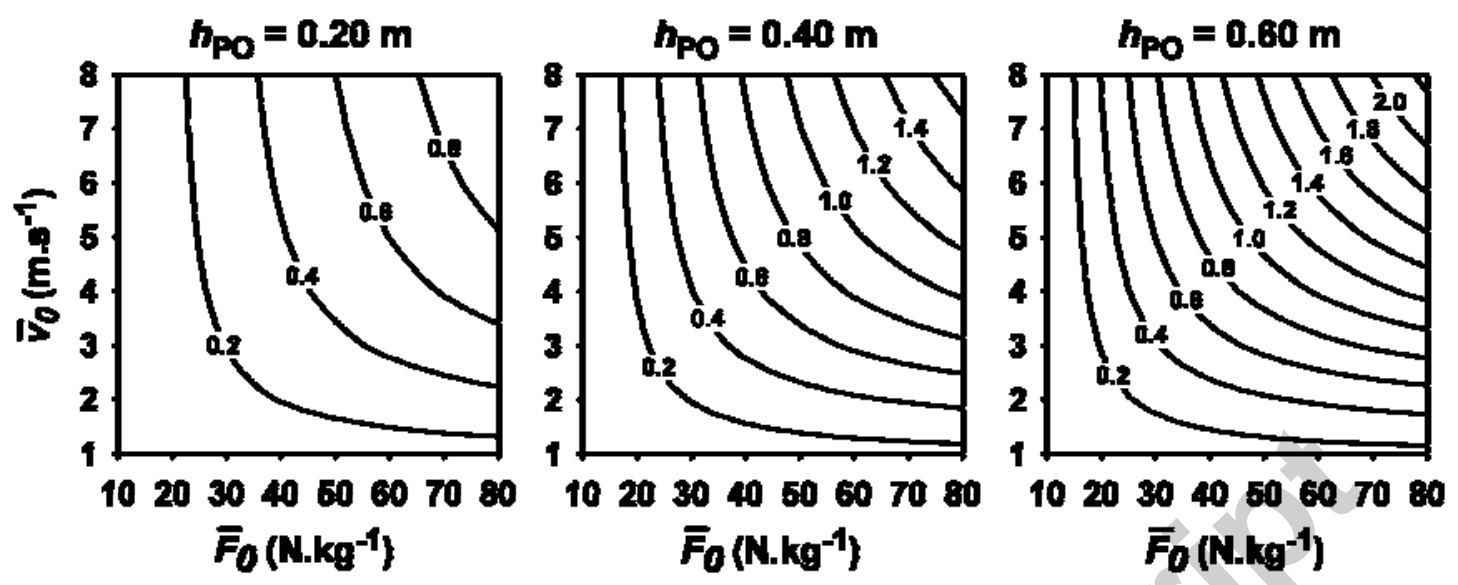

